\title{
IncRNAs as potential molecular biomarkers in the clinicopathology and prognosis of cholangiocarcinoma: a systematic review and meta-analysis
}

This article was published in the following Dove Medical Press journal: OncoTargets and Therapy

\author{
Kangfu Dai ${ }^{1,2}$ \\ Jing Quan' \\ Fangli Yan' \\ Xinghan Jin' \\ Xiang Pan' \\ Xiaorui Song' \\ Shijie Zhang' \\ Qingqi Ren² \\ Jikui Liu',2 \\ Xiaoping Liu'1,2 \\ 'Clinical College, Peking University \\ Shenzhen Hospital, Anhui Medical \\ University, Hefei, Anhui, 230032, \\ P.R. China; ${ }^{2}$ Department of \\ HepatoBiliary and Pancreatic Surgery, \\ Peking University Shenzhen Hospital, \\ Shenzhen, Guangdong 518036, China
}

Correspondence: Xiaoping Liu; Jikui Liu Department of HepatoBiliary and Pancreatic Surgery, Peking University Shenzhen Hospital, I 20 Lianhua Road, Shenzhen, Guangdong 5I8036, China Email ninliu@163.com; 185657978I6@I63.com

\begin{abstract}
Background: Cholangiocarcinoma (CCA) is the second most common fatal primary hepatobiliary malignant carcinoma, characterized by early invasion and extremely poor outcomes. It is therefore necessary to identify a novel biomarker to better diagnose CAA and predict its prognosis. Recently, emerging evidence has revealed that some lncRNAs play an important role in the tumorigenesis and progression of CAA. In order to support this search for novel diagnostic and prognostic biomarkers for CAA, we conducted a meta-analysis to analyze the published association between lncRNA expression and its clinical value in CAA.

Methods: Eligible studies were pooled and analyzed according to our inclusion and exclusion criteria after a comprehensive literature search. Stata 14.0 software was used to analyze the data from relevant studies and to construct a forest plot. Different effect sizes were selected for the meta-analysis.

Results: In total, 24 publications were included in this meta-analysis. After review of their fulltext, 16 articles studied the association between IncRNAs and clinicopathological characteristics, 2 discussing diagnosis and 16 discussing prognosis. Our results showed that overexpression of CCAT1 was significantly correlated with tumor stage (I + II vs III + IV) (OR, 4.99; 95\% CI 2.77-8.99; $P<0.001)$ and lymph node metastasis in CCA (OR, 4.75; 95\% CI 2.65-8.52; $P<0.001)$. Furthermore, elevated CCAT IncRNA family expression predicted a shorter overall survival (HR, 2.09; 95\% CI 1.17-3.00; $P<0.001$ ), especially CCAT2. Upregulation of CCAT2 was also obviously associated with tumor stage in CCA (OR, 5.29; 95\% CI 2.64-10.58; $P=0.001$ ). Conclusion: This is the first meta-analysis to assess the relationship between expression of IncRNAs and the clinical values of patients with CCA. IncRNAs can function as potential molecular biomarkers of the clinicopathology and prognosis of CCA.
\end{abstract}

Keywords: lncRNA, cholangiocarcinoma, clinicopathological characteristics, diagnosis, prognosis

\section{Introduction}

Cholangiocarcinoma (CCA) originates in the epithelium of hepatic biliary trees and is the second most common fatal primary hepatobiliary malignant carcinoma. ${ }^{1}$ According to recent epidemiological data, the incidence and mortality of CCA in the world has been increasing rapidly over the past decades. ${ }^{2,3}$ However, due to the lack of a specific clinical presentation and effective diagnostic systems for CCA, most of CCA patients are diagnosed at advanced stages. ${ }^{2,4}$ Additionally, due to tumor resistance to 
traditional chemotherapy and radiotherapy, surgery is currently the most effective treatment for $\mathrm{CCA} .{ }^{3}$ As a result, the prognosis of patients with CCA is extremely poor, with a high rate of recurrence, and a 5-year survival rate of only $5 \%{ }^{5,6}$ For these reasons, identifying new therapeutic targets and novel biomarkers associated with CCA diagnosis and prognosis is very important to improve outcomes for those with this disease.

lncRNAs are RNA molecules transcribed without functional open reading frames and are $>200$ nucleotides in length. ${ }^{7}$ They function in various biological processes mostly by binding with miRNAs as sponges or interacting with proteins, including those active in cell proliferation, migration, invasion, and apoptosis. ${ }^{8}$ Recently, numerous studies indicated that aberrant expression of lncRNAs was involved in tumorigenesis and cancer progression, including CCA. ${ }^{7-10}$ Emerging evidence also demonstrated that some lncRNAs are associated with the diagnosis and prognosis of CAA. ${ }^{8,11,12}$

However, due to limitations related to small sample sizes and various experimental protocols, a single study on these topics may be inaccurate and are therefore insufficiently powered to inform solid conclusions. Thus, the aim of the present study was to systematically analyze all studies of CAA to assess the potential clinical value of lncRNAs in CAA. Here, we identify the relationship between lncRNAs expression and three different clinical outcomes (clinicopathological characteristics, diagnosis, and prognosis).

\section{Methods}

\section{Search strategy}

Two of the authors (KD and JQ) independently searched several databases, including PubMed, Embase, the Cochrane Library, China National Knowledge Internet (CNKI), Wanfang and Weipu database, for studies on lncRNAs and CCA. The literature was searched up to September 13, 2018. The search

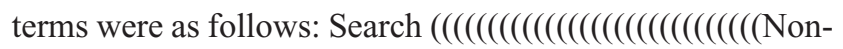
coding RNA, Long[Title/Abstract]) OR lncRNA[Title/ Abstract]) OR Long ncRNA[Title/Abstract]) OR ncRNA, Long[Title/Abstract]) OR RNA, Long Non-Translated[Title/ Abstract]) OR Long Non-Translated RNA[Title/Abstract]) OR Non-Translated RNA, Long[Title/Abstract]) OR RNA, Long Non Translated[Title/Abstract]) OR Long Non-Coding RNA[Title/Abstract]) OR Long Non Coding RNA[Title/ Abstract]) OR Non-Coding RNA, Long[Title/Abstract]) OR RNA, Long Non-Coding[Title/Abstract]) OR Long NonProtein-Coding RNA[Title/Abstract]) OR Long Non Protein Coding RNA[Title/Abstract]) OR Non-Protein-Coding RNA, Long[Title/Abstract]) OR RNA, Long Non-Protein-
Coding[Title/Abstract]) OR Long Noncoding RNA[Title/ Abstract]) OR RNA, Long Untranslated[Title/Abstract]) OR Long Untranslated RNA[Title/Abstract]) OR Untranslated RNA, Long[Title/Abstract]) OR Long ncRNAs[Title/ Abstract]) OR ncRNAs, Long[Title/Abstract]) OR Long Intergenic Non-Protein Coding RNA[Title/Abstract]) OR Long Intergenic Non Protein Coding RNA[Title/ Abstract]) OR LincRNAs[Title/Abstract]) OR LINC RNA[Title/Abstract])) OR “RNA, Long Noncoding"[Mesh])) AND $((()(()(()((()(C h o l a n g i o c a r c i n o m a s[$ Title/Abstract] $))$ OR Cholangiocellular Carcinoma[Title/Abstract]) OR Carcinoma, Cholangiocellular[Title/Abstract]) OR Carcinomas, Cholangiocellular[Title/Abstract]) OR Cholangiocellular Carcinomas[Title/Abstract]) OR Extrahepatic Cholangiocarcinoma[Title/Abstract]) OR Cholangiocarcinoma, Extrahepatic[Title/Abstract]) OR Cholangiocarcinomas, Extrahepatic[Title/Abstract]) OR Extrahepatic Cholangiocarcinomas[Title/Abstract]) OR Intrahepatic Cholangiocarcinoma[Title/Abstract]) OR Cholangiocarcinoma, Intrahepatic[Title/Abstract]) OR Cholangiocarcinomas, Intrahepatic[Title/Abstract]) OR Intrahepatic Cholangiocarcinomas[Title/Abstract])) OR “Cholangiocarcinoma”[Mesh]).

\section{Inclusion and exclusion criteria}

The inclusion criteria were as follows: 1) patients diagnosed with CCA by histopathology; 2) the expression level of lncRNAs divided into high and low, and the correlation between lncRNAs expression and clinicopathological features were detailed; 3 ) the relationship between IncRNA expression and survival outcome, hazard risk (HR), 95\% CI, or $P$-value, and Kaplan-Meier curves were outlined; 4$)$ the expression of IncRNAs was detected in the tissue or serum, and sufficient data on sensitivity, specificity, and sample size were presented.

The exclusion criteria were as follows: 1) non-human studies; 2) letters, case reports, commentaries, conference abstracts, or review articles; 3 ) articles unrelated to lncRNA and CCA; 4) insufficient data for extraction; 5) HRs calculated using multiple lncRNAs; 6) studies focused on genetic polymorphisms or modification of lncRNAs.

\section{Data extraction and quality assessment}

Two independent authors (KD and JQ) extracted the information from the included literature using a predefined template based on the reporting checklists of PRISMA:13 1 ) the first author's last name and publication year; 2) the type of IncRNA, study population, region, sample number, follow-up time (months), and detection methods; 3 ) clinicopathological 
features: age, gender, tumor size, histological grade, tumor stage, lymph node metastasis, distant metastasis, carbohydrate antigen 199, alpha-fetoprotein (AFP), and hepatitis B virus (HBV) infection; 4) $\mathrm{HRs,} 95 \% \mathrm{CI}$, and $P$-value for survival analysis. If HRs were directly available, we collected these from the original studies, otherwise these data were indirectly extracted from Kaplan-Meier curves according to the method of Tierney et $\mathrm{al}^{14}$ or we asked the authors for these data; and 5) diagnostic data were included: sensitivity, specificity, and area under curve (AUC).

We assessed the quality of all the included diagnostic studies according to the Quality Assessment of Diagnostic Accuracy Studies- $2^{15}$ criteria and used the Newcastle-Ottawa Scale ${ }^{16}$ to assess the quality of the selected prognostic studies (scores $>5$ was regarded as high quality).

\section{Statistical analysis}

Heterogeneity among articles was assessed with Higgin's $I^{2}$ statistic. $I^{2}>50 \%$ indicated statistically significant heterogeneity. A fixed-effects or random-effects model was applied to evaluate the relationship between IncRNAs expression and survival outcomes. A fixed-effects model was used when heterogeneity among studies was not obvious. Otherwise, a random-effects model was used. ${ }^{17,18}$ A different effect size was selected for each meta-analysis: 1) that of clinicopathological features analyzed OR and associated 95\% CI. 2) In the diagnostic meta-analysis, sensitivity, specificity, and AUC were used. 3) The prognostic meta-analysis employed HR and associated $95 \% \mathrm{CI}$ for each study to estimate the survival outcomes associated with the expression of $\operatorname{lncRNA}$. HR $>1$ was regarded as the worse survival for the group with elevated lncRNA expression. ${ }^{18}$ Stata 14.0 software (StataCorp LP, College Station, TX, USA) was used to analyze study data and construct the forest plot. $P<0.05$ was considered to be statistically significant.

\section{Results \\ Study identification and characteristics}

As shown in the search flowchart (Figure 1), 88 articles in total were retrieved from PubMed, Embase, Cochrane, and three Chinese databases (China Knowledge Resource Integrated, Wanfang, and Weipu databases). Further, 20 articles
Records retrieved from PubMed ( $n=26)$, Embase $(n=52)$ Cochrane $(n=0)$, CNKI $(n=4)$, Wanfan $(n=3)$, and Weipu $(n=3)$
Additional records identified through other sources $(n=0)$

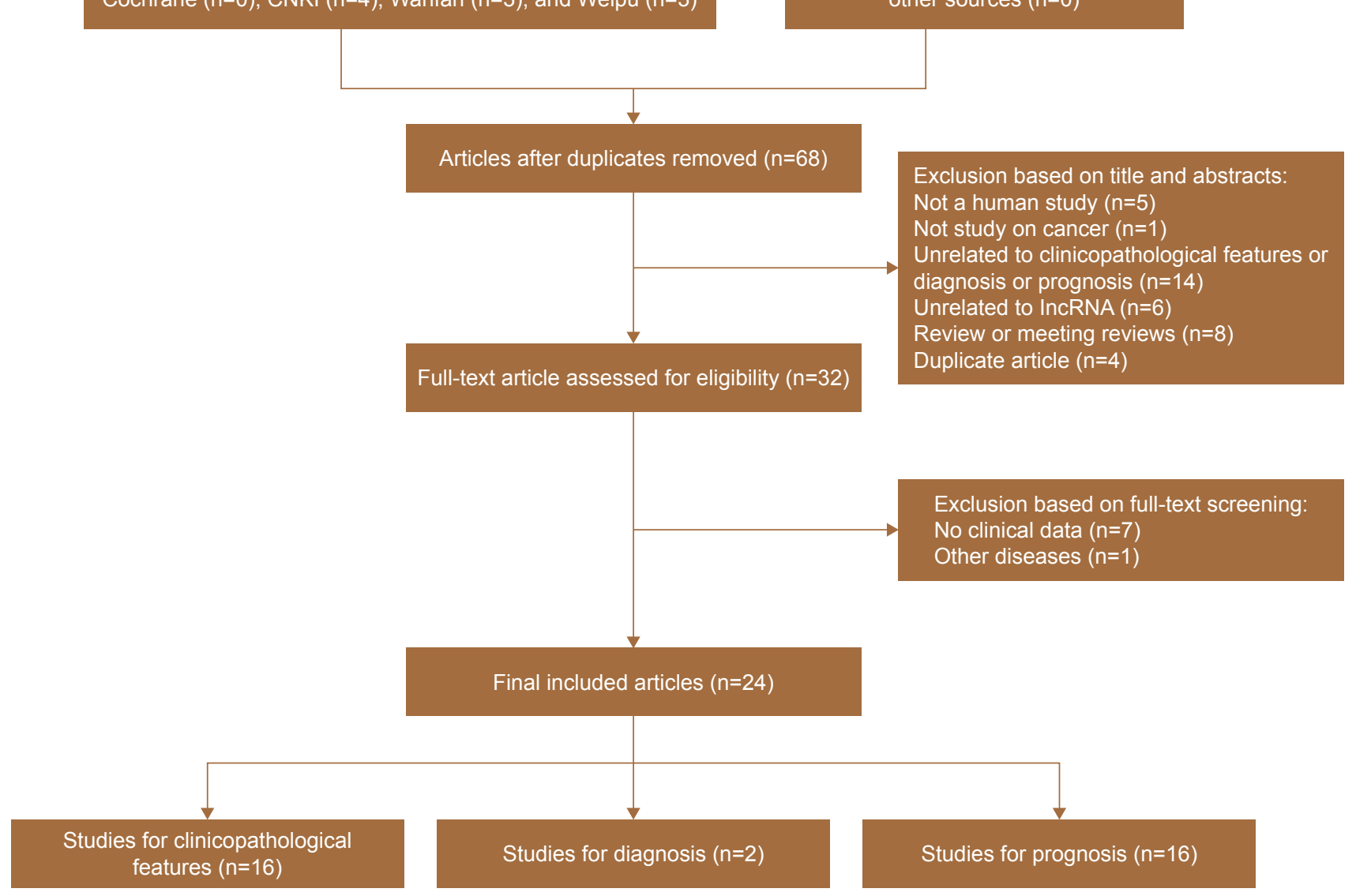

Figure I The study selection process. 
Table I The association between IncRNAs and clinicopathological features

\begin{tabular}{|c|c|c|c|c|c|c|c|}
\hline Study & Region & IncRNA & $\begin{array}{l}\text { Simple size } \\
\text { (n) }\end{array}$ & $\begin{array}{l}\text { Detection } \\
\text { method }\end{array}$ & Expression & Age & $\begin{array}{l}\text { Gender } \\
\text { (P-value) }\end{array}$ \\
\hline Wang et al, $2016^{20}$ & China & $\mathrm{HIO}$ & 72 & RT-qPCR & Up & 0.954 & 0.538 \\
\hline Ma et al, $2015^{22}$ & China & CPSI-ITI & 31 & RT-qPCR & Up & 0.862 & 0.693 \\
\hline \multirow[t]{4}{*}{ Lv et al, $2017^{19}$} & \multirow[t]{4}{*}{ China } & EMPI-008 & \multirow[t]{4}{*}{72} & \multirow[t]{4}{*}{ RT-qPCR } & Down & 0.89 & 0.412 \\
\hline & & ATF3-008 & & & Down & 0.388 & 0.431 \\
\hline & & RCOR3-0I3 & & & Up & 0.917 & 0.359 \\
\hline & & TMEM63A-005 & & & Up & 0.941 & 0.826 \\
\hline Xu et al, $2017^{8}$ & China & CCATI & 91 & RT-qPCR & $U_{p}$ & 0.413 & 0.875 \\
\hline Zhang et al, $2017^{24}$ & China & CCATI & 120 & RT-qPCR & Up & 0.938 & 0.407 \\
\hline Xu et al, $2017^{25}$ & China & PANDAR & 67 & RT-qPCR & Up & 0.307 & 0.457 \\
\hline Lu et al, $2017^{11}$ & China & AFAPI-ASI & 56 & RT-qPCR & Up & 0.768 & 1.000 \\
\hline Zhang et al, $2017^{24}$ & China & Linc01296 & 57 & RT-qPCR & Up & 0.553 & 0.789 \\
\hline Tan et al, $2017^{12}$ & China & MALATI & 62 & RT-qPCR & $U_{p}$ & 0.602 & 0.799 \\
\hline Xu et al, $2017^{25}$ & China & UCAI & 68 & RT-qPCR & Up & 0.621 & 0.807 \\
\hline Li et al, $2017^{\prime \prime}$ & China & Sox2ot & 58 & RT-qPCR & Up & 0.301 & $0.57 I$ \\
\hline Xu et al, $2017^{25}$ & China & $\mathrm{HIO}$ & 56 & RT-qPCR & Up & 0.282 & 0.596 \\
\hline Xia et al, $2018^{27}$ & China & CRNDE & 118 & RT-qPCR & Up & 0.276 & 0.306 \\
\hline Bai et al, $2018^{28}$ & China & CСAT2 & 106 & RT-qPCR & $U_{p}$ & 0.204 & 0.620 \\
\hline Xu et al, $2018^{29}$ & China & ССАT2 & 60 & RT-qPCR & Up & 0.435 & 0.796 \\
\hline Xu et al, $2018^{29}$ & China & HOTAIR & 70 & RT-qPCR & Up & 0.624 & 0.544 \\
\hline
\end{tabular}

Abbreviations: RT-qPCR, real-time quantitative PCR; CAI99, carbohydrate antigen 199; AFP, alpha-fetoprotein; HBV, hepatitis B virus; NA, not available.

were excluded as duplicates. After reviewing the remaining titles and abstracts carefully, 36 studies were removed, including 5 non-human studies; 1 study not on CCA; 14 studies unrelated to clinicopathological features, diagnosis, or prognosis; 6 studies without lncRNAs; 8 reviews or meeting reviews; and 4 more duplicate articles. Finally, 24 studies were deemed eligible for meta-analysis upon further review of the full-text article, including 16 discussing on clinicopathological characteristics, 2 on diagnosis, and 16 on prognosis.

\section{Clinicopathological characteristics}

Herein, 14 lncRNAs were described in 13 included studies on clinicopathological characteristics. As shown in the Table 1, except EMP1-008 ${ }^{19}$ and ATF3-008, ${ }^{19}$ almost all lncRNAs were upregulated in CCA, including H19, ${ }^{20,21}$ CPS1-IT1, ${ }^{22}$ RCOR3-013, ${ }^{19}$ TMEM63A-005, ${ }^{19}$ CCAT1,${ }^{23,24}$ PANDAR, ${ }^{8}$ AFAP1-AS $1,{ }^{11}$ Linc01296, ${ }^{6}$ MALAT $1,{ }^{12}$ UCA $1,{ }^{25}$ Sox $20 t,{ }^{26}$ CRNDE,${ }^{27}$ CCAT2, ${ }^{28,29}$ and HOTAIR. ${ }^{30}$ All studies indicated that lncRNAs were not significantly associated with patient age, gender, and HBV infection status. Few studies demonstrated that the expression levels of lncRNAs were associated with AFP, except H19. ${ }^{20}$ Only one study indicated that CPS1-IT1 expression was significantly related to carcinoembryonic antigen (CEA), ${ }^{22}$ and seven studies reported that six lncRNAs were significantly correlated with tumor size, including H19, ${ }^{20,21}$ AFAP1-AS1, ${ }^{11}$ Linc01296, ${ }^{6}$ MALAT $1,{ }^{12}$ CRNDE, ${ }^{25}$ and HOTAIR.${ }^{30}$

Only four studies found that lncRNAs were significantly associated with histological grade of CCA, while 13 studies claimed that lncRNAs were significantly correlated with tumor grade of CCA. Interestingly, Table 1 shows that AFAP1-AS1 and CCAT2 expressions were significantly related to vascular invasion. ${ }^{11,28}$ Additionally, ten studies demonstrated that $\operatorname{lncRNAs}$ were significantly associated with lymph node metastasis, while three studies reported that IncRNAs were significantly correlated with distant metastasis. Table 1 also revealed that H19, CCAT1, and CCAT2 were all detected in two articles. Therefore, we combined these two studies with a total of six groups by constructing two-by-two tables. However, H19 could not be further analyzed due to insufficient data. Based on our meta-analysis of these articles describing CCAT1, the relationship between upregulation of CCAT1 and tumor stage (I + II vs III + IV) was indeed significant (OR, 4.99; 95\% CI $2.77-8.99 ; P<0.001)$. Furthermore, overexpression CCAT1 was significantly correlated with the lymph node metastasis in CCA (OR, 4.75; 95\% CI 2.65-8.52; $P<0.001)$ (Figure 2). 


\begin{tabular}{|c|c|c|c|c|c|c|c|c|}
\hline $\begin{array}{l}\text { Tumor size } \\
(\mathrm{cm})\end{array}$ & $\begin{array}{l}\text { Histological } \\
\text { grade (I-IV) }\end{array}$ & Tumor stage & $\begin{array}{l}\text { Vascular } \\
\text { invasion }\end{array}$ & $\begin{array}{l}\text { Lymph node } \\
\text { metastasis }\end{array}$ & $\begin{array}{l}\text { Distant } \\
\text { metastasis }\end{array}$ & CA199 & AFP & HBV \\
\hline $0.001(5)$ & 0.091 & 0.062 & 0.144 & 0.005 & 0.092 & 0.924 & 0.031 & NA \\
\hline 0.677 (NA) & NA & NA & 0.642 & 0.045 & NA & 0.044 & NA & NA \\
\hline $0.314(5)$ & NA & NA & NA & NA & 0.012 & 0.819 & 0.603 & 0.914 \\
\hline $0.304(5)$ & NA & NA & NA & NA & 0.001 & 0.321 & 0.482 & 0.484 \\
\hline $0.379(5)$ & NA & NA & NA & NA & 0.03 & 0.923 & 0.319 & 0.926 \\
\hline $0.271(5)$ & NA & NA & NA & NA & 0.955 & 0.141 & 0.094 & 0.807 \\
\hline NA & 0.636 & 0.005 & NA & 0.01 & NA & 0.490 & NA & 0.909 \\
\hline NA & 0.612 & $<0.01$ & NA & $<0.01$ & NA & NA & NA & NA \\
\hline NA & 0.014 & 0.034 & 0.794 & 0.004 & NA & 0.221 & $\mathrm{NA}$ & 0.788 \\
\hline $0.031(5)$ & 0.003 & 0.013 & 0.011 & 0.540 & 0.177 & NA & NA & 1.000 \\
\hline $0.003(3)$ & NA & 0.024 & NA & 0.031 & NA & NA & NA & NA \\
\hline $0.042(3)$ & NA & 0.037 & NA & 0.037 & NA & NA & NA & NA \\
\hline NA & 0.307 & 0.004 & NA & 0.027 & NA & NA & $\mathrm{NA}$ & NA \\
\hline NA & 0.849 & 0.007 & 0.203 & 0.031 & NA & 0.067 & NA & 0.813 \\
\hline 0.029 (3) & 0.783 & 0.015 & 0.245 & 0.105 & NA & 0.173 & NA & 0.418 \\
\hline 0.001 (5) & 0.046 & 0.050 & 0.742 & NA & 0.114 & 0.172 & 0.374 & NA \\
\hline $0.326(5)$ & 0.040 & $<0.001$ & $<0.001$ & NA & NA & 0.327 & NA & 0.919 \\
\hline $0.228(3)$ & 0.414 & 0.010 & NA & 0.019 & NA & NA & $\mathrm{NA}$ & NA \\
\hline $0.028(3)$ & 0.609 & 0.021 & 0.468 & 0.805 & NA & 0.609 & NA & 0.789 \\
\hline
\end{tabular}

Study ID

Age

Jiang 2017

Zhang 2017

Subtotal $\left(I^{2}=0.0 \%, P=0.561\right)$

Gender

Jiang 2017

Zhang 2017

Subtotal $\left(I^{2}=0.0 \%, P=0.508\right)$

TNM stage

Jiang 2017

Zhang 2017

Subtotal $\left(I^{2}=22.4 \%, P=0.256\right)$

Lymph node metastasis

Jiang 2017

Zhang 2017

Subtotal $\left(I^{2}=43.0 \%, P=0.185\right)$

Overall $\left(I^{2}=79.7 \%, P=0.000\right)$

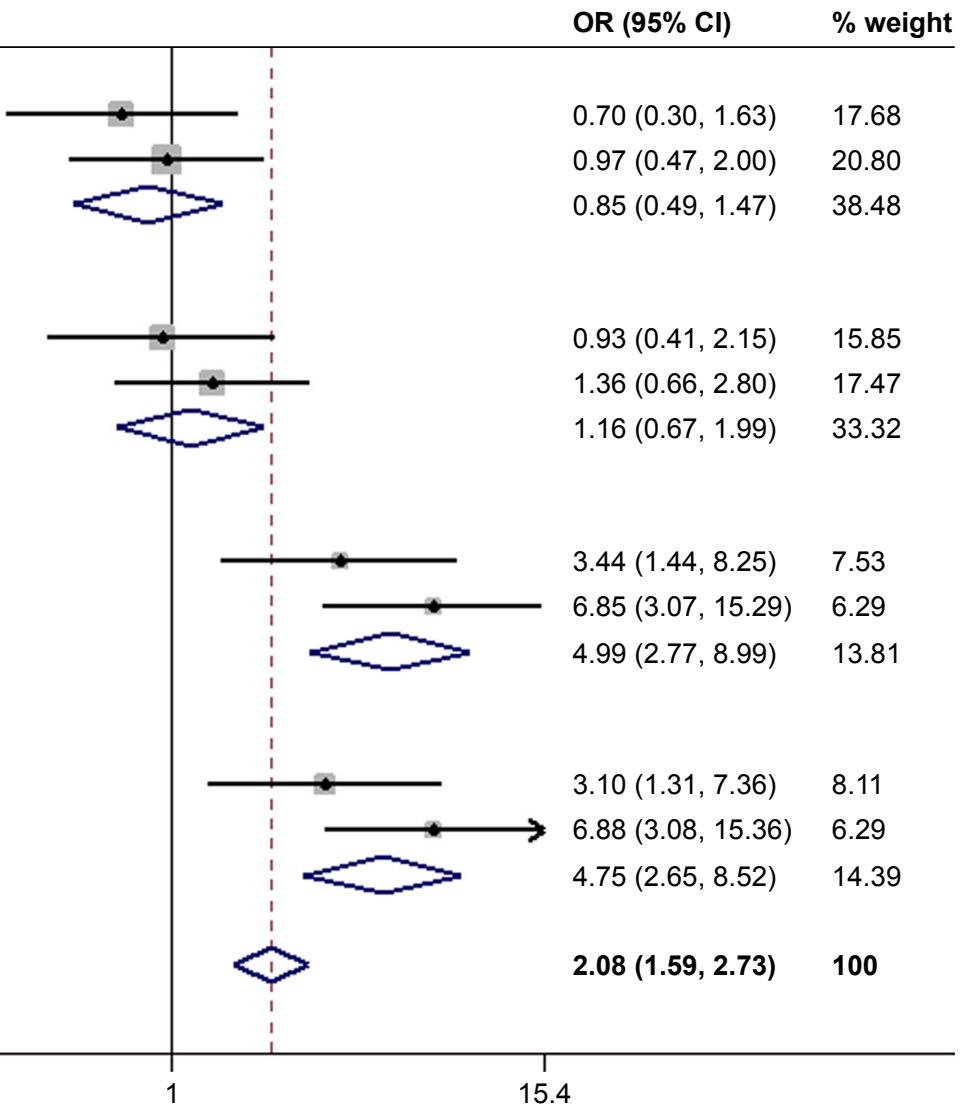

Figure 2 Forest plots of studies evaluating the odds ratio of upregulated CCATI expression and the clinicopathology of cholangiocarcinoma patients. 


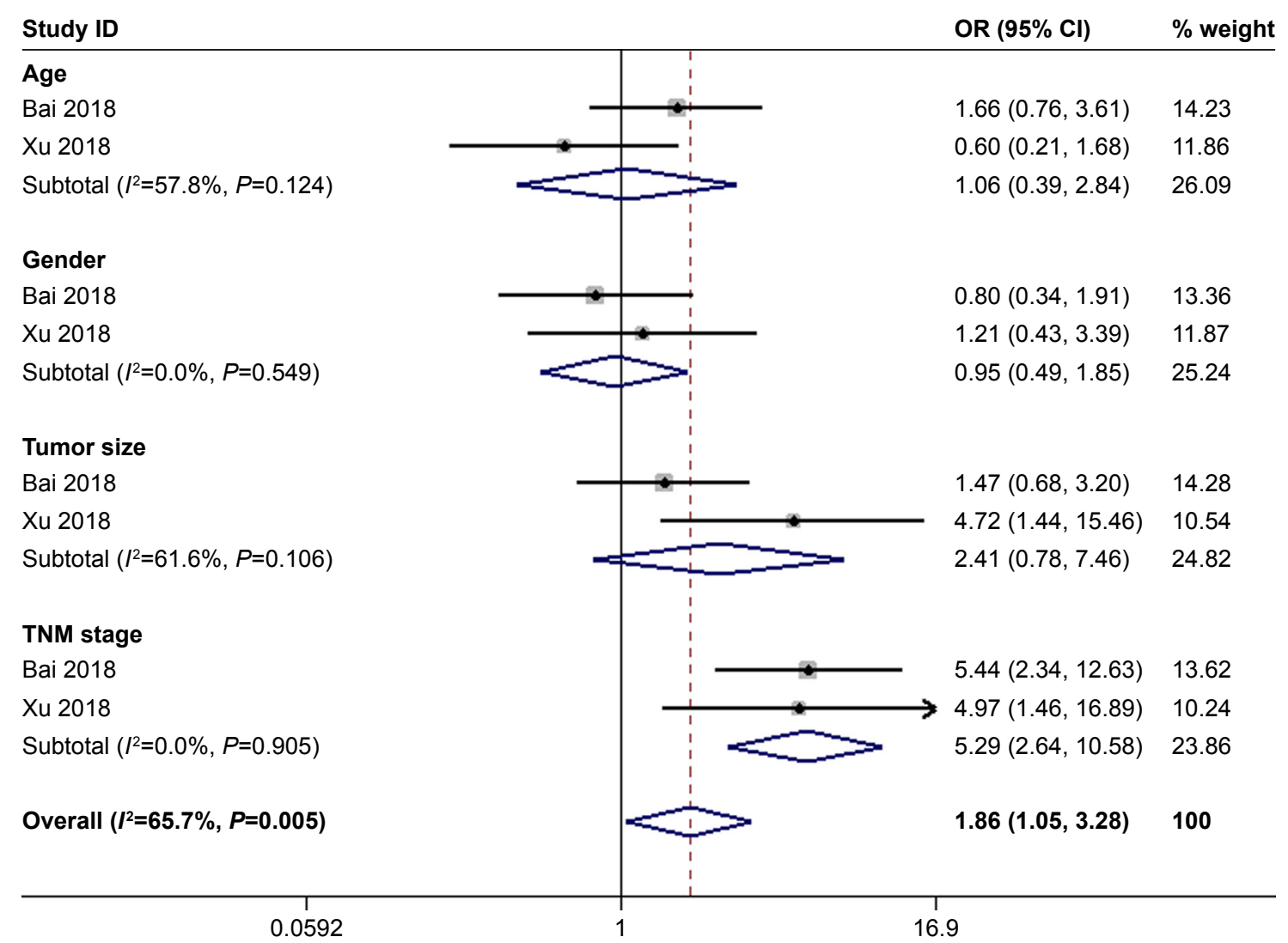

Figure 3 Forest plots of studies evaluating the odds ratio of upregulated CCAT2 expression and the clinicopathology of cholangiocarcinoma patients. Note: Weights are from random-effects analysis.

Similarly, the result about CCAT2 also indicated that upregulation of CCAT2 was obviously associated with tumor stage in CCA (OR, 5.29; 95\% CI 2.64-10.58; $P=0.001$ ) (Figure 3). However, dysfunction of CCAT2 was not related with age, gender, and tumor size $(P>0.05)$.

\section{Diagnosis}

Only two studies about three lncRNAs were included in this topical analysis. All these lncRNAs were upregulated in different detected samples using real-time quantitative PCR (RT-qPCR). Jiang et al indicated that CCAT1 acted as a potential biomarker for the diagnosis of CCA with relative high sensitivity (81.80\%) and specificity (74.50\%). ${ }^{23}$ Furthermore, Ge et al detected ENST00000517758.1 and ENST00000588480.1 in bile samples and found they were both potential biomarkers for the diagnosis of CCA. ${ }^{31}$ The main characteristics of these two studies are presented in Table 2.

\section{Prognosis}

Briefly, 16 different lncRNAs were described in the 16 included studies on prognosis. The characteristics of these eligible studies are presented in Table 3 . The lncRNA expression of all samples was detected from tissues by RT-qPCR. This analysis included 585 patients with high expression and 522 patients with low expression in total. Interestingly, all of these lncRNAs were overexpressed in CCA, with elevated expression levels associated with poor prognosis, including CPS1-IT1, ${ }^{22}$ CCAT $1,{ }^{23}$ TUG1, ${ }^{32}$ PANDAR, ${ }^{8}$ AFAP1-AS1, ${ }^{11}$ LINC01296, ${ }^{6}$ MALAT1, ${ }^{12}$ UCA1, ${ }^{25}$ Sox2ot, ${ }^{26}$

Table 2 Summary of IncRNAs used as diagnostic biomarkers of cholangiocarcinoma

\begin{tabular}{|c|c|c|c|c|c|c|c|c|c|c|}
\hline \multirow[t]{2}{*}{ Study } & \multirow[t]{2}{*}{ Region } & \multirow[t]{2}{*}{ IncRNA } & \multirow[t]{2}{*}{ Expression } & \multirow[t]{2}{*}{ SE (\%) } & \multirow[t]{2}{*}{ SP (\%) } & \multirow[t]{2}{*}{ AUC } & \multicolumn{2}{|c|}{ Sample size } & \multirow{2}{*}{$\begin{array}{l}\text { Detected } \\
\text { sample }\end{array}$} & \multirow[t]{2}{*}{ QUADAS } \\
\hline & & & & & & & Case & Control & & \\
\hline $\begin{array}{l}\text { Xu et al, } \\
2017^{8}\end{array}$ & China & CCTAI & Up & 81.80 & 74.50 & 0.831 & 91 & 91 & Tissue & 7 \\
\hline \multirow{2}{*}{$\begin{array}{l}\text { Ge et al, } \\
2017^{31}\end{array}$} & \multirow[t]{2}{*}{ China } & ENST000005I I758.I & Up & - & - & 0.613 & \multirow[t]{2}{*}{35} & \multirow[t]{2}{*}{56} & \multirow[t]{2}{*}{ Bile sample } & \multirow[t]{2}{*}{5} \\
\hline & & ENST00000588480.I & Up & 62.90 & 73.20 & 0.680 & & & & \\
\hline
\end{tabular}

Abbreviations: SE, sensitivity; SP, specificity; AUC, area under curve; QUADAS, Quality Assessment of Diagnostic Accuracy Studies. 


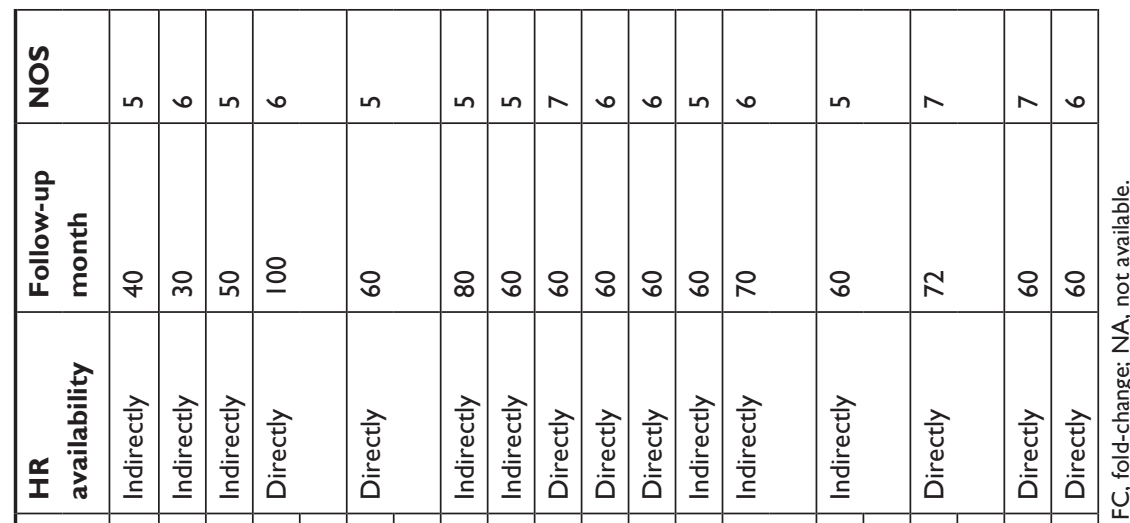

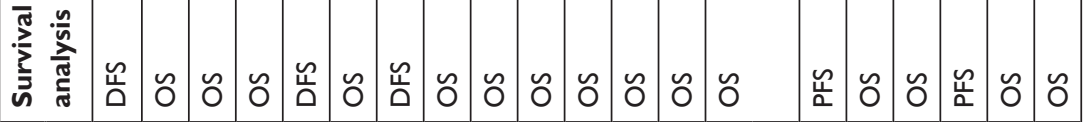

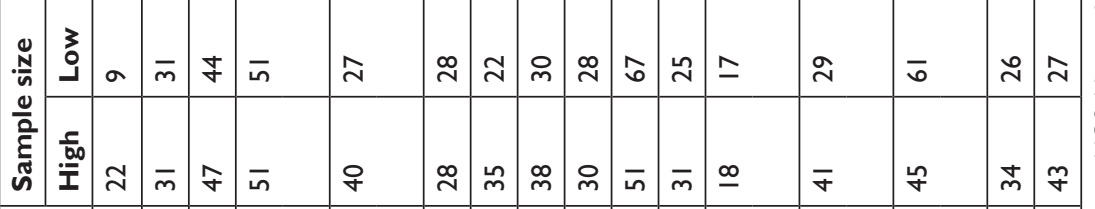

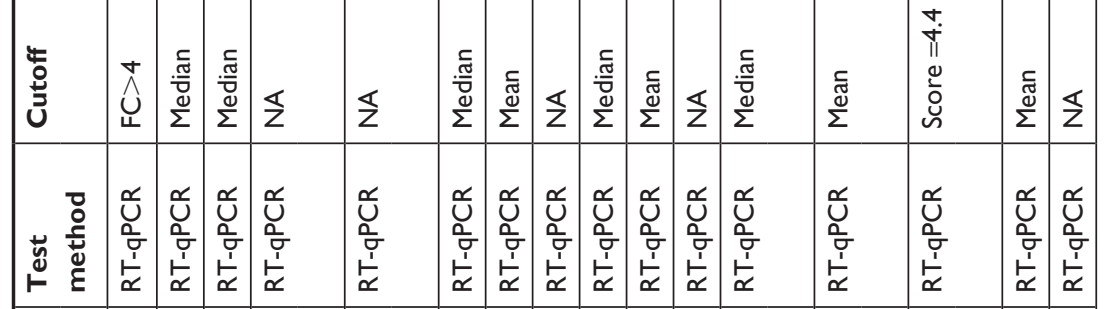

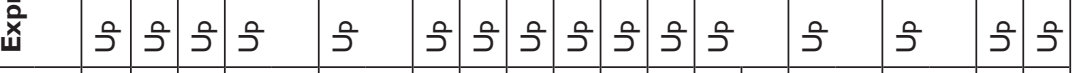




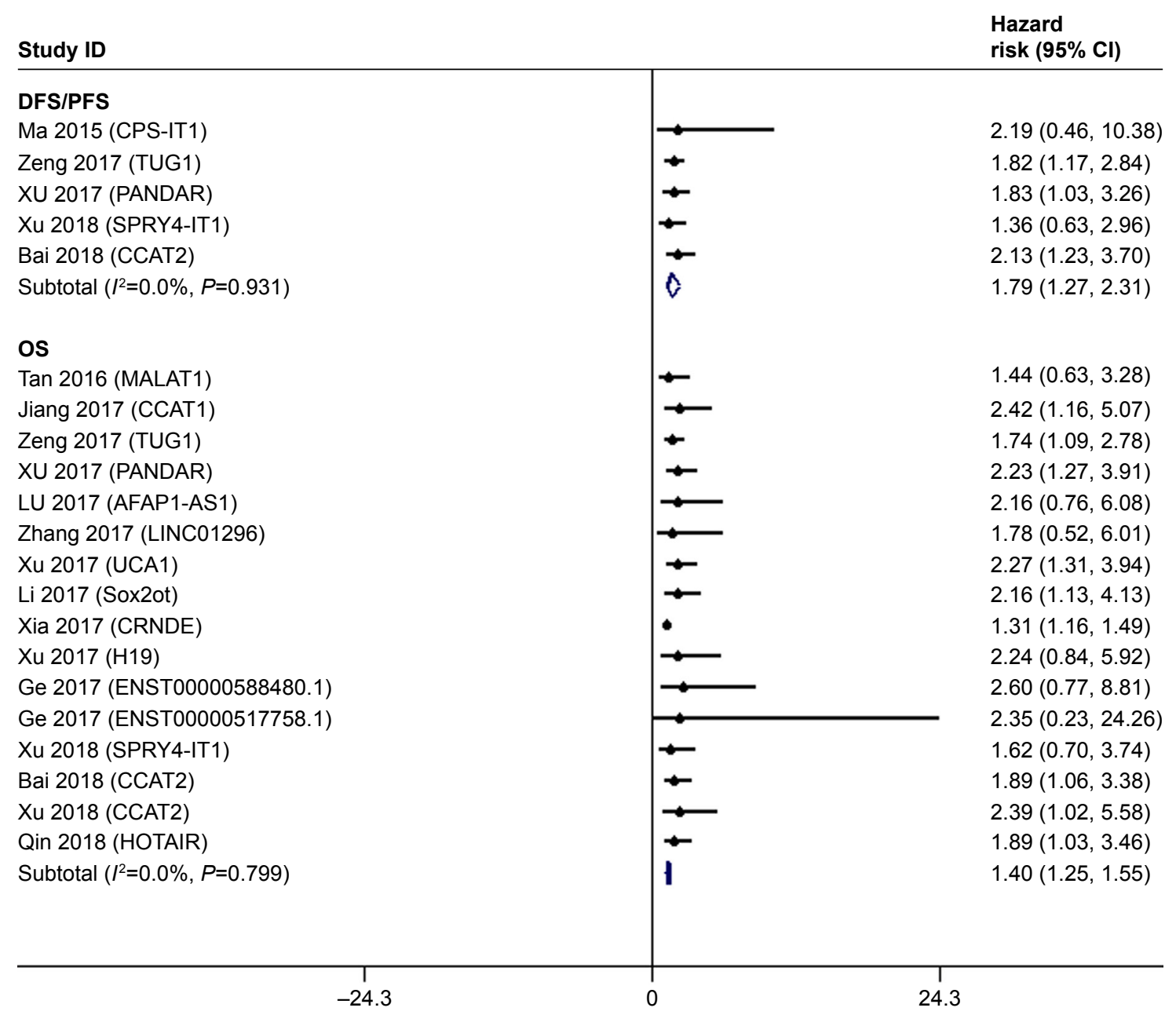

Figure 4 Display of the hazard risk (HR) of IncRNAs and overall survival (OS) in cholangiocarcinoma patients. Abbreviations: DFS, disease-free survival; PFS, progression-free survival.

CRNDE, ${ }^{27} \mathrm{H} 19,{ }^{21}$ SPRY4-IT1, ${ }^{33}$ ENST00000588480.1, ${ }^{31}$ ENST00000517758.1, ${ }^{31}$ CCAT2, ${ }^{28,34}$ and HOTAIR ${ }^{30}$ (Figure 4). Among them, Sox $2 \mathrm{ot}^{26}$ had the highest HR of 2.936, while CRNDE ${ }^{27}$ displayed the lowest HR with 1.309.

Among these 16 lncRNAs, the CCAT family was investigated in more than one study. Thus, we further analyzed the relationship between the expression of CCAT gene family and overall survival (OS; Figure 5). A fixed-effects model was used due to the lack of significant heterogeneity in the CCAT family $\left(I^{2}=0.0 \%, P=0.867\right)$. As a result, high CCAT family expression predicted short OS (HR, 2.09; 95\% CI $1.17-3.00 ; P<0.001)$. By further subgroup analysis, overexpression of CCAT2 was obviously associated with poor OS (HR, 2.00; 95\% CI 0.96-3.03; $P<0.001$ ). Publication bias could not be assessed because of the small size of our study.

\section{Discussion}

CCA is still a deadly threat to human health due to its early invasion and metastatic characteristics and poor prognosis.
According to relevant reports, the global incidence of CCA has clearly increased during the past decades, especially in Asia. ${ }^{3,35}$ However, current therapeutics for CAA are unsatisfactory and so novel biomarkers to diagnose CAA and predict its prognosis are urgently needed. Recently, there has been emerging evidence showing that some lncRNAs play an important role in the tumorigenesis and progression of CAA. In order to codify some of these novel biomarkers for CCA, we conducted this systematic review and meta-analysis. As a result, this metaanalysis is the first to systematically analyze the association between lncRNA expression and their clinical value in CCA.

In terms of association with clinicopathological features, H19, ${ }^{20,21}$ CPS1-IT1, ${ }^{22}$ RCOR3-013, ${ }^{19}$ TMEM63A-005, ${ }^{19}$ CCAT $1,{ }^{23,24}$ PANDAR, ${ }^{8}$ AFAP1-AS $1,{ }^{11}$ Linc01296, ${ }^{6}$ MALAT $1,{ }^{12} \mathrm{UCA} 1,{ }^{25}$ Sox2ot, ${ }^{26} \mathrm{CRNDE},{ }^{27} \mathrm{CCAT} 2,{ }^{28,34}$ and HOTAIR ${ }^{30}$ were overexpressed in CCA, while EMP1-008 ${ }^{19}$ and ATF3-008 ${ }^{19}$ were downregulated. All 1ncRNAs were not significantly associated with patient age, gender, and HBV infection. 


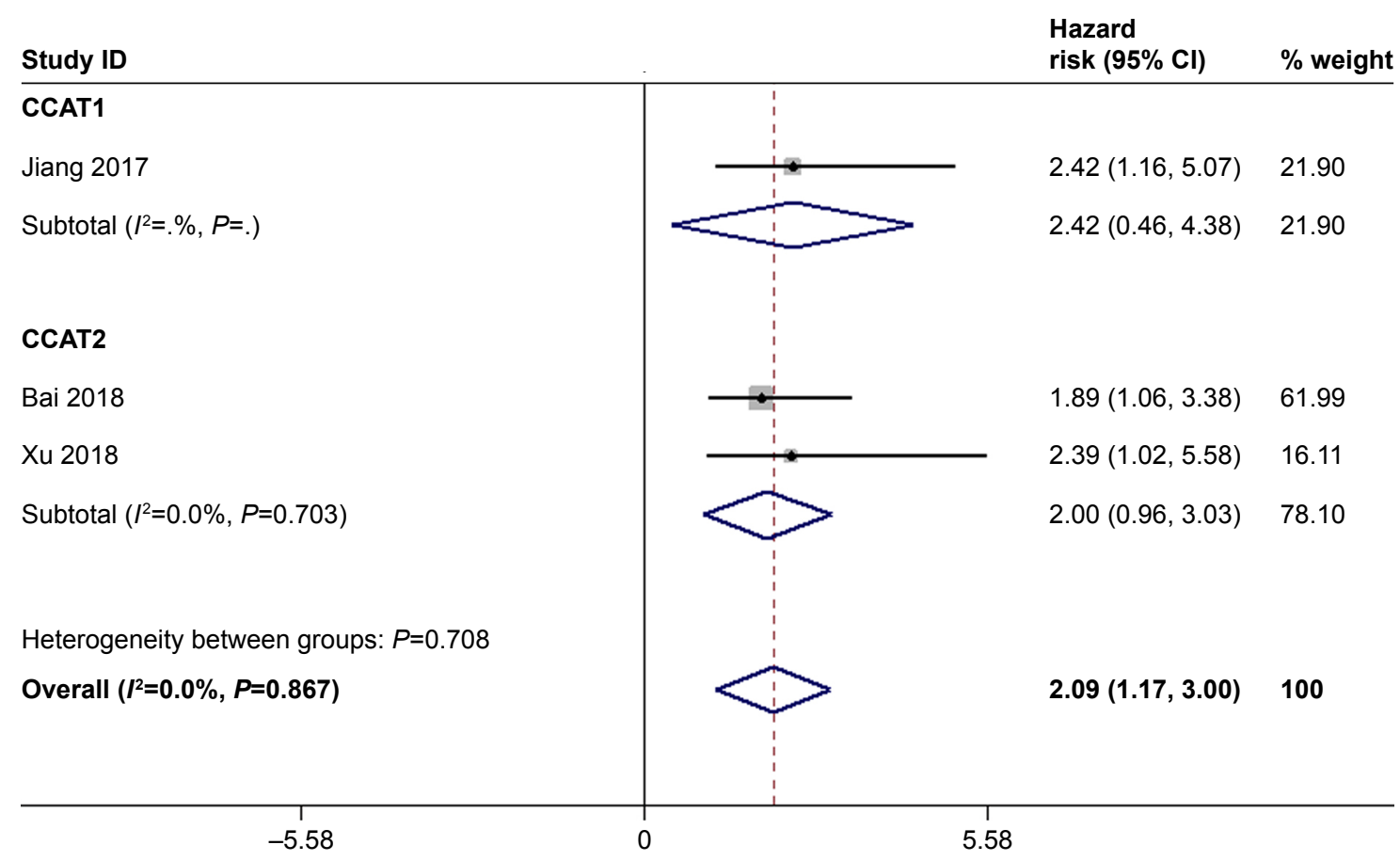

Figure 5 Forest plots of studies evaluating the hazard ratios (HRs) of upregulated CCAT family expression and the overall survival (OS) of cholangiocarcinoma patients.

It is well-known that AFP and CEA play an important role in the diagnosis of CCA. ${ }^{36}$ However, studies did not indicate that dysregulation of lncRNAs was significantly related to AFP and CEA. Only two studies indicated that H19 expression was obviously associated with AFP, ${ }^{20}$ and CPS1-IT1 expression was significantly related to CEA. ${ }^{22}$ The reason for association of $\mathrm{H} 19$ with $\mathrm{AFP}$ is that the $A f p$ and $H 19$ genes are regulated by $A f r l$, which was first identified in 1977 using persistent AFP serum levels. ${ }^{37,38}$ There is no direct evidence for the relationship between CPS1-IT1 and CEA.

Similarly, we found that six lncRNAs were significantly correlated with tumor size, including H19, ${ }^{20,21}$ AFAP1-AS1, ${ }^{11}$ Linc01296, ${ }^{6}$ MALAT $1,{ }^{12}$ CRNDE, ${ }^{25}$ CCAT2, ${ }^{28,34}$ and HOTAIR ${ }^{30}$ However, the correlation remained uncertain due to the different evaluation criteria for tumor size. For example, in the study about association between $\mathrm{H} 19$ expression and tumor size, Wang et $\mathrm{al}^{20}$ selected $5 \mathrm{~cm}$ as the criterion for tumor size, while Xu et $\mathrm{al}^{21}$ used $3 \mathrm{~cm}$ as the threshold of tumor size. The reason why some lncRNAs were significantly correlated with tumor size is still uncertain. Moreover, most IncRNAs were significantly correlated with tumor grade of CCA, which meant these lncRNAs were correlated with the progression of CCA.

Ten studies demonstrated that lncRNAs were significantly associated with lymph node metastasis. Among them, we conducted a meta-analysis to further analyze the relationship between CCAT1 expression and clinical features. Our results indicated that CCAT1 expression was significantly correlated with tumor stage and lymph node metastasis in CCA. The above-mentioned results were also reported in other tumors, such as breast cancer ${ }^{39}$ and esophageal squamous cell carcinoma. ${ }^{40}$ However, Arunkumar et al found that CCAT1 was not obviously associated with tumor stage and the lymph node metastasis in oral squamous cell carcinomas $(P>0.05){ }^{41}$ Similarly, another meta-analysis was also performed to analyze the relationship between CCAT2 expression and clinical features. As a result, CCAT2 indicated that upregulation of CCAT2 was obviously associated with tumor stage in CCA.

In our analysis of the prognostic value of lncRNAs, all included studies demonstrated that the overexpression of $\operatorname{lncRNAs}$ was associated with a poor prognosis. Among these lncRNAs, the CCAT family was investigated in more than one study. After analysis, the results showed that high expression of CCAT family members predicted shorter OS, especially CCAT2. Our result is consistent with the previous findings, while more research is needed to verify this conclusion due to the small sample size of this study. ${ }^{42,43}$

This study was not without limitations. Firstly, the number of studies included was small. Most lncRNAs appeared only once within the incorporated studies and few lncRNAs appeared in more than two different studies, which influenced heterogeneity. Secondly, due to the lack of survival data, we extracted HR and 95\% CI values from a Kaplan-Meier 
curve according to Tierney et al methodology, which might also cause potential heterogeneity. Thirdly, because all the included studies were from China, these results might not be applicable to other ethnicities, such as Caucasians. Next, different cutoff values and follow-up end points were used among the included studies, leading to potential heterogeneity. Finally, studies with positive results were more likely to be published, which may result in an exaggeration of the clinical values of lncRNAs in CAA. In spite of these limitations, our study effectively confirmed the important role of various lncRNA expressions in CAA and encouraged researchers to explore the underlying mechanisms in the future.

So far, there are no reports about the application of lncRNA as a biomarker in clinical practice. However, some lncRNAs have been shown to be more sensitive and specific than existing markers. ${ }^{44,45}$ So we believe that some lncRNAs will be identified for use in the clinic as biomarkers in the future.

\section{Conclusion}

Taken together, our results show that some specific lncRNAs are significantly associated with clinical value in CAA patients. Among them, upregulation of CCAT1 is significantly associated with tumor stage (I + II vs III + IV) and lymph node metastasis for CCA. Overexpression of CCAT2 is obviously related with tumor stage. Additionally, high expression of CCAT family members predicted shorter OS, especially with regards to CCAT2. However, further large-scale and high-quality studies should be included to confirm our findings and to verify the clinical value of lncRNAs in CCA.

\section{Data sharing statement}

The analyzed data sets generated during the study are available from the corresponding author on reasonable request.

\section{Acknowledgments}

This work is was supported by Sanming Project of Medicine in Shenzhen (No SZSM201612021), Special Fundation for Science and Technology Development of Guangdong Province (No 2017B090904010), and Scientific Research Project of Health and Family Planning Commission of Shenzhen Municipality (No SZXJ2018086).

\section{Author contributions}

Kangfu Dai and Jing Quan are co-first authors. KD and JQ designed this study and were involved in data collection, data analysis, and manuscript writing. FY, XP, and XJ contributed to data collection and data analysis. XS and SZ contributed to data analysis. QR was involved in the language editing of the manuscript. All authors contributed to data analysis, drafting and revising the article, gave final approval to be published, and agree to be accountable for all aspects of the work.

\section{Disclosure}

The authors report no conflicts of interest in this work.

\section{References}

1. de Groen PC, Gores GJ, LaRusso NF, Gunderson LL, Nagorney DM. Biliary tract cancers. N Engl J Med. 1999;341(18):1368-1378.

2. Razumilava N, Gores GJ. Cholangiocarcinoma. Lancet. 2014;383(9935): 2168-2179.

3. Khan SA, Davidson BR, Goldin RD, et al. Guidelines for the diagnosis and treatment of cholangiocarcinoma: an update. Gut. 2012;61(12): $1657-1669$.

4. Khan SA, Thomas HC, Davidson BR, Taylor-Robinson SD. Cholangiocarcinoma. Lancet. 2005;366(9493):1303-1314.

5. Bridgewater J, Galle PR, Khan SA, et al. Guidelines for the diagnosis and management of intrahepatic cholangiocarcinoma. $J$ Hepatol. 2014;60(6):1268-1289

6. Zhang D, Li H, Xie J, et al. Long noncoding RNA LINC01296 promotes tumor growth and progression by sponging miR-5095 in human cholangiocarcinoma. Int J Oncol. 2018;52(6):1777-1786.

7. Zhang A, Zhang J, Kaipainen A, Lucas JM, Yang H. Long non-coding RNA: a newly deciphered "code" in prostate cancer. Cancer Lett. 2016; 375(2):323-330.

8. Xu Y, Jiang X, Cui Y. Upregulated long noncoding RNA PANDAR predicts an unfavorable prognosis and promotes tumorigenesis in cholangiocarcinoma. Onco Targets Ther. 2017;10:2873-2883.

9. Schmitt AM, Chang HY. Long noncoding RNAs in cancer pathways. Cancer Cell. 2016;29(4):452-463.

10. Huarte M. The emerging role of lncRNAs in cancer. Nat Med. 2015; 21(11):1253-1261.

11. Lu X, Zhou C, Li R, Deng Y, Zhao L, Zhai W. Long noncoding RNA AFAP1-AS1 promoted tumor growth and invasion in cholangiocarcinoma. Cell Physiol Biochem. 2017;42(1):222-230.

12. Tan X, Huang Z, Li X. Long non-coding RNA MALAT1 interacts with miR-204 to modulate human hilar cholangiocarcinoma proliferation, migration, and invasion by targeting CXCR4. J Cell Biochem. 2017; 118(11):3643-3653.

13. Moher D, Liberati A, Tetzlaff J, Altman DG; PRISMA Group. Preferred reporting items for systematic reviews and meta-analyses: the PRISMA statement. PLoS Med. 2009;6(7):e1000097.

14. Tierney JF, Stewart LA, Ghersi D, Burdett S, Sydes MR. Practical methods for incorporating summary time-to-event data into meta-analysis. Trials. 2007;8:16.

15. Whiting PF, Rutjes AW, Westwood ME, et al. QUADAS-2: a revised tool for the quality assessment of diagnostic accuracy studies. Ann Intern Med. 2011;155(8):529-536.

16. Lo CK, Mertz D, Loeb M. Newcastle-Ottawa Scale: comparing reviewers' to authors' assessments. BMC Med Res Methodol. 2014;14:45.

17. Gu L, Li H, Chen L, et al. MicroRNAs as prognostic molecular signatures in renal cell carcinoma: a systematic review and meta-analysis. Oncotarget. 2015;6(32):32545-32560.

18. Chen J, Chen Y, Gu L, et al. IncRNAs act as prognostic and diagnostic biomarkers in renal cell carcinoma: a systematic review and metaanalysis. Oncotarget. 2016;7(45):74325-74336.

19. Lv L, Wei M, Lin P, et al. Integrated mRNA and lncRNA expression profiling for exploring metastatic biomarkers of human intrahepatic cholangiocarcinoma. Am J Cancer Res. 2017;7(3):688-699.

20. Wang J, Xie H, Ling Q, et al. Coding-noncoding gene expression in intrahepatic cholangiocarcinoma. Transl Res. 2016;168:107-121. 
21. Xu Y, Wang Z, Jiang X, Cui Y. Overexpression of long noncoding RNA H19 indicates a poor prognosis for cholangiocarcinoma and promotes cell migration and invasion by affecting epithelial-mesenchymal transition. Biomed Pharmacother. 2017;92:17-23.

22. Ma SL, Li AJ, Hu ZY, Shang FS, Wu MC. Co-expression of the carbamoyl-phosphate synthase 1 gene and its long non-coding RNA correlates with poor prognosis of patients with intrahepatic cholangiocarcinoma. Mol Med Rep. 2015;12(6):7915-7926.

23. Jiang XM, Li ZL, Li JL, et al. IncRNA CCAT1 as the unfavorable prognostic biomarker for cholangiocarcinoma. Eur Rev Med Pharmacol Sci. 2017;21(6):1242-1247.

24. Zhang S, Xiao J, Chai Y, et al. IncRNA-CCAT1 promotes migration, invasion, and EMT in intrahepatic cholangiocarcinoma through suppressing miR-152. Dig Dis Sci. 2017;62(11):3050-3058.

25. Xu Y, Yao Y, Leng K, et al. Long non-coding RNA UCA1 indicates an unfavorable prognosis and promotes tumorigenesis via regulating AKT/GSK-3 $\beta$ signaling pathway in cholangiocarcinoma. Oncotarget. 2017;8(56):96203-96214.

26. Li Z, Li J, Ji D, et al. Overexpressed long noncoding RNA Sox2ot predicts poor prognosis for cholangiocarcinoma and promotes cell proliferation and invasion. Gene. 2018;645:131-136.

27. Xia XL, Xue D, Xiang TH, et al. Overexpression of long non-coding RNA CRNDE facilitates epithelial-mesenchymal transition and correlates with poor prognosis in intrahepatic cholangiocarcinoma. Oncol Lett. 2018;15(4):4105-4112.

28. Bai JG, Tang RF, Shang JF, Qi S, Yu GD, Sun C. Upregulation of long non-coding RNA CCAT2 indicates a poor prognosis and promotes proliferation and metastasis in intrahepatic cholangiocarcinoma. Mol Med Rep. 2018;17(4):5328-5335.

29. Xu Y, Yao Y, Qin W, et al. Long non-coding RNA CCAT2 promotes cholangiocarcinoma cells migration and invasion by induction of epithelial-to-mesenchymal transition. Biomed Pharmacother. 2018;99:121-127.

30. Qin W, Kang P, Xu Y, et al. Long non-coding RNA HOTAIR promotes tumorigenesis and forecasts a poor prognosis in cholangiocarcinoma. Sci Rep. 2018;8(1):12176.

31. Ge X, Wang Y, Nie J, et al. The diagnostic/prognostic potential and molecular functions of long non-coding RNAs in the exosomes derived from the bile of human cholangiocarcinoma. Oncotarget. 2017;8(41):69995-70005.

32. Zeng B, Ye H, Chen J, et al. IncRNA TUG1 sponges miR-145 to promote cancer progression and regulate glutamine metabolism via Sirt3/GDH axis. Oncotarget. 2017;8(69):113650-113661.
33. Xu Y, Yao Y, Jiang X, et al. SP1-induced upregulation of lncRNA SPRY4-IT1 exerts oncogenic properties by scaffolding EZH2/LSD1/ DNMT1 and sponging miR-101-3p in cholangiocarcinoma. J Exp Clin Cancer Res. 2018;37(1):81.

34. Xu Y, Yao Y, Qin W, Zhong X, Jiang X, Cui Y. Long non-coding RNA CCAT2 promotes cholangiocarcinoma cells migration and invasion by induction of epithelial-to-mesenchymal transition. Biomed Pharmacother. 2018;99:121-127.

35. Zhu AX. Future directions in the treatment of cholangiocarcinoma. Best Pract Res Clin Gastroenterol. 2015;29(2):355-361.

36. Li Y, Li DJ, Chen J, et al. Application of joint detection of AFP, CA19-9, CA125 and CEA in identification and diagnosis of cholangiocarcinoma. Asian Pac J Cancer Prev. 2015;16(8):3451-3455.

37. Belayew A, Tilghman SM. Genetic analysis of alpha-fetoprotein synthesis in mice. Mol Cell Biol. 1982;2(11):1427-1435.

38. Pachnis V, Belayew A, Tilghman SM. Locus unlinked to alphafetoprotein under the control of the murine raf and Rif genes. Proc Natl Acad Sci U S A. 1984;81(17):5523-5527.

39. Zhang XF, Liu T, Li Y, Li S. Overexpression of long non-coding RNA CCAT1 is a novel biomarker of poor prognosis in patients with breast cancer. Int J Clin Exp Pathol. 2015;8(8):9440-9445.

40. Zhang E, Han L, Yin D, et al. H3K27 acetylation activated-long non-coding RNA CCAT1 affects cell proliferation and migration by regulating SPRY4 and HOXB13 expression in esophageal squamous cell carcinoma. Nucleic Acids Res. 2017;45(6):3086-3101.

41. Arunkumar G, Murugan AK, Prasanna Srinivasa Rao H, Subbiah S, Rajaraman R, Munirajan AK. Long non-coding RNA CCAT1 is overexpressed in oral squamous cell carcinomas and predicts poor prognosis. Biomed Rep. 2017;6(4):455-462.

42. Jing X, Liang H, Cui X, Han C, Hao C, Huo K. Long noncoding RNA CCAT2 can predict metastasis and a poor prognosis: a meta-analysis. Clin Chim Acta. 2017;468:159-165.

43. Fan Y-H, Fang H, Ji C-Xing, et al. Long noncoding RNA CCAT2 can predict metastasis and poor prognosis: a meta-analysis. Clin Chim Acta. 2017;466:120-126.

44. Xie Y, Zhang Y, du L, et al. Circulating long noncoding RNA act as potential novel biomarkers for diagnosis and prognosis of non-small cell lung cancer. Mol Oncol. 2018;12(5):648-658.

45. Wang WW, Zhou XL, Song YJ, Yu CH, Zhu WG, Tong YS. Combination of long noncoding RNA MALAT1 and carcinoembryonic antigen for the diagnosis of malignant pleural effusion caused by lung cancer. Onco Targets Ther. 2018;11:2333-2344.
OncoTargets and Therapy

\section{Publish your work in this journal}

OncoTargets and Therapy is an international, peer-reviewed, open access journal focusing on the pathological basis of all cancers, potential targets for therapy and treatment protocols employed to improve the management of cancer patients. The journal also focuses on the impact of management programs and new therapeutic agents and protocols on

\section{Dovepress}

patient perspectives such as quality of life, adherence and satisfaction. The manuscript management system is completely online and includes a very quick and fair peer-review system, which is all easy to use. Visit http://www.dovepress.com/testimonials.php to read real quotes from published authors. 